\title{
Discrimination of haploid and diploid males of Bombus terrestris (Hymenoptera; Apidae) based on wing shape
}

\author{
Maxence Gerard ${ }^{1}$, Denis Michez ${ }^{1}$, Denis Fournier ${ }^{2}$, Kevin Maebe $^{3}$, Guy Smagghe ${ }^{3}$, \\ Jacobus C. Biesmeijer ${ }^{4}$, Thibaut De Meulemeester ${ }^{1}$ \\ ${ }^{1}$ Laboratory of Zoology, University of Mons, Place du Parc 20, 7000, Mons, Belgium \\ ${ }^{2}$ Evolutionary Biology and Ecology, Université libre de Bruxelles, Brussels, Belgium \\ ${ }^{3}$ Department of Crop Protection, Faculty of Bioscience Engineering, Ghent University, Coupure links 653, 9000, Ghent, \\ Belgium \\ ${ }^{4}$ Naturalis Biodiversity Center, Darwinweg 2, PO Box 9517, 2300RA, Leiden, The Netherlands
}

Received 23 April 2014 - Revised 19 January 2015 - Accepted 2 February 2015

\begin{abstract}
Presence of diploid males in wild bees reflects inbreeding and provides information about the health status of a colony or population. Detection of diploid males, and discrimination from haploid males and workers, has, however, been limited to molecular diagnostics. Here we present a novel method based on differences in wing shape, e.g., venation patterns in wings. The method is easy to apply and results, for Bombus terrestris, in very high discrimination success. Possible applications of the method are discussed.
\end{abstract}

\section{Bombus / decline / diploid males / wing shape / geometric morphometrics}

\section{INTRODUCTION}

There is accumulating evidence of recent declines in both wild and domesticated bees (Biesmeijer et al. 2006; Potts et al. 2010; Cameron et al. 2011). Among the potential drivers of these declines are landscape modification and fragmentation, intensive use of agrochemicals, pathogen infections, competition with alien species, climate change, and interactions between all of these (Potts et al. 2010; Winfree 2010). Independent of which driver is responsible, reduced populations experience several threats such as loss of genetic diversity and risk of inbreeding

Electronic supplementary material The online version of this article (doi:10.1007/s13592-015-0352-3) contains supplementary material, which is available to authorized users.

Corresponding author: T. De Meulemeester, thibaut.demeulemeester@naturalis.nl; thibaut.demeulemeester@umons.ac.be Manuscript editor: James Nieh as a result of non-random mating (Ellis et al. 2006; Davis et al. 2010). These threats are particularly problematic in haplodiploid species such as bees (Zayed and Packer 2005; Zayed 2009). These species have a single locus sex determination system where diploids are females and haploids are males (Whiting 1933; Crozier 1971). However, low genetic diversity, due to inbreeding depression, can lead to diploids being homozygous at this locus and therefore developing into males (Duchateau et al. 1994). More than 80 Hymenoptera species produce diploid males (Van Wilgenburg et al. 2006; Darvill et al. 2012). While in some eusocial species (e.g., Apis mellifera) diploid male larvae are killed by workers before becoming an adult (Woyke 1963), in bumble bees homozygote diploid larvae may develop into unviable or effectively sterile males.

Production of viable diploid males has a strong negative effect on bumble bees population maintenance by reducing the fitness of (1) the colonies producing the diploid males and (2) the queens 
mating with diploid males. Indeed, after mating with a brother, half of the queen's diploid eggs, which normally develop into females, develop into males (Duchateau et al. 1994). As a consequence, the colony produces only half the normal quantity of workers from the start and thus will remain rather small in size, with low production of new queens. Second, as most bumble bee species are monandrous (even known polyandrous species mate with a frequency close to one; Estoup et al. 1996; Payne et al. 2003; Takahashi et al. 2008) and diploid males produce diploid sperm, queens mating with diploid males produce $100 \%$ of sterile triploid offspring leading to the inbred strain having much lower fitness than outbred colonies. In wild populations, this is known as the "diploid male extinction vortex" (Zayed and Packer 2005). In this vortex, populations are reduced, e.g., due to habitat fragmentation, which leads to decreased allelic diversity, increased inbreeding, and the production of diploid males so that the extinction risk of the population increases. Nevertheless the haplodiploid system could select negatively against the deleterious alleles and thereby balance the costs of inbreeding and low genetic diversity (Schmid-Hempel et al. 2007). Some studies have tried to quantify the presence of diploid males in wild populations. In Japanese Bombus florilegus , $28 \%$ of the males were diploid (Takahashi et al. 2008), whereas diploid males were also frequent in fragmented populations of British Bombus muscorum (Darvill et al. 2006). Because diploid male production occurs under conditions of severe inbreeding, the presence of diploid males can be used as an indicator for population (or colony) health and can be used to assess the viability of bee populations (Zayed et al. 2004).

Detection of low genetic diversity or increased inbreeding within a population requires specialized, often invasive, and very costly genetic methods, such as flow cytometry or microsatellite analysis (Gadau et al. 2001; Maebe et al. 2013, 2014). Use of morphological, instead of genetic, characters could provide an alternative, but has not been developed until now. Even though morphological methods have been developed to detect A. mellifera diploid drone larvae (Santomauro and Engels 2002) or adults based on testes and genital hooks (Herrmann et al. 2005), fast and effective morphometrics identifiers are still lacking for wild bees.

In this study, we developed and tested wing morphometric identifiers for bumble bee diploid males. Our study focuses on the bumble bee Bombus terrestris, a well-studied and easily reared bumble bee. Wings were analyzed by geometric morphometric procedures, which quantify and analyze the overall shape of a structure (Bookstein 1991; Rohlf and Marcus 1993; Zelditch et al. 2004; Adams et al. 2013). Morphometric studies of bees have focused on wing shape and venation because wings are flat, rigid 2D structures with many homologous landmarks that are diagnostic at multiple taxonomic levels (e.g., Francoy et al. 2008; Michez et al. 2009; De Meulemeester et al. 2012; Dewulf et al. 2014). Our method is cheap, fast, and renders the possibility of assessing male ploidy not only in freshly collected specimens but also in pin-mounted museum specimens for which biomolecular analysis is often impeded due to inadequate preservation.

\section{MATERIALS AND METHODS}

\subsection{Material and ploidy determination}

Three colonies of $B$. terrestris obtained from bee breeder Biobest N.V. were artificially reared in a controlled climate room at $+28 / 30{ }^{\circ} \mathrm{C}$ and $60 \%$ relative humidity at the laboratory of Zoology in Mons (UMons, $\mathrm{BE})$. Only one of the colonies produced both queens $(n=11)$ and males $(n=21)$ of first generation. The newly emerged queens and males of this colony were placed in a "flight cage" to force brother-sister mating. These mated queens were used to produce second-generation inbred colonies. During 2 months, the mated queens overwintered following the rearing method of Lhomme et al. (2013). Hibernated queens were activated with $\mathrm{CO}_{2}$ narcosis (Roseler 1985) and were placed in small boxes with two workers and a cocoon to maximize the probability of colony initiation (Kwon et al. 2003).

The 11 mated queens of the first generation initiated colonies, producing a total of 62 workers and 41 males of second generation. The degree of ploidy of these males was assessed by flow cytometry using a PA-I flow cytometer (PARTECC); Partec GmbH, Munster, 
Germany). Head cells were extracted in DNA-staining solution containing DAPI as the DNA dye. For this purpose, bumble bees were first killed by deep freezing (at $-80^{\circ} \mathrm{C}$ ) and the head was placed each in $1 \mathrm{ml}$ of the DNA-staining solution. Processing, cell cycle analysis and quantification of flow cytometric data were performed according to Cournault and Aron (2008). Both wings were removed for geometric morphometric analyses.

The total sample of specimens for the analysis included 30 workers, 21 haploid males, and 10 matedqueens of the first generation, and 48 workers, 26 diploid males, and 10 haploid males of the second generation.

\subsection{Geometric morphometrics}

The right and left forewings of all 145 specimens were photographed using an Olympus SZH10 microscope coupled with a Nikon D200 camera. Among the 290 wings, 274 were suitable for morphometrics analyses (from 78 workers, nine queens, 28 haploid males, 22 diploid males). Left wings were mirror-reflected to achieve landmark homology. Photographs were input to tps-UTILS 1.56 (Rohlf 2013a) and both right and left wing shapes were captured by digitizing twodimensional Cartesian coordinates of 18 landmarks (Figure 1) on wing veins with tps-DIG v2.17 (Rohlf 2013b).

The 274 landmark configurations were scaled, translated, and rotated against the consensus configuration using the GLS Procrustes superimposition method to remove all non-shape differences and to separate the size and shape components of the form (Rohlf and Slice 1990; Bookstein 1991). The superimposition was performed using $\mathrm{R}$ functions of the package geomorph (Adams and Otárola-Castillo 2013).

The aligned landmark configurations were projected into the Euclidean space tangent to the curved Kendall's shape space to aid further statistical analyses. The correlation coefficient between the Procrustes distances in the shape space and the Euclidean distances in the linear tangent space equaled 1.00. This indicates that the curvature of the shape space around our data was negligible (Rohlf 1999). The least-squares regression slope through the origin (0.999) and the correlation coefficient between the two distances were calculated with tps-SMALL v1.25 (Rohlf 2013c).

\subsection{Shape discrimination of castes and diploid males}

We evaluated potential differences between wings of the four groups (i.e., queen, worker, haploid male, diploid male) in their size and shape. Significance of size differences among the different castes and between haploid and diploid males was tested by analysis of variance (ANOVA) of mean centroid sizes by specimen (CS). CS is defined as the square root of the sum of squared distance between all landmarks and their centroid (Zelditch et al. 2004). Preliminary verification reveals that wing CS is related to the length of the radial cell (distance from the first wing submarginal cell venule to the wing radial cell apex), which was already used as a proxy for total body length (Medler 1962).

\subsubsection{Discrimination of single wings}

A between-group PCA was performed to visualize shape variation between the four groups (Figure 2). Compared to traditional PCA, between-group PCA tends to maximize variation between groups by projecting the greatest variance between group means along the first principal component, the second greatest variance on the second component, and so on. Unlike discriminant analyses, scores of the specimens are projected in a Euclidean space that does not affect relations or distances between specimens. Influence of size on shape analyses was tested by correlation coefficient between CS and components of the bgPCA.

Discrimination among the different castes and between haploid and diploid males was also assessed by linear discriminant analysis (LDA) of the aligned configuration of landmarks. The effectiveness of the LDA for discriminating groups was assessed by the percentages of wings correctly classified to their original group (i.e., hit ratio, HR) in a leave-one-out cross-validation procedure based on the posterior probabilities of assignment. Given the observed scores of a wing, the posterior probability equals the probability of the wing to belong to one group compared to all others. The wing is consequently assigned to the group for which the posterior probability is the highest (Huberty and Olejnik 2006). As the right and left forewings of all specimens were included in our analyses, therefore a pseudoreplica effect could occur in the cross-validation procedure. To avoid overfitting of HR, we simultaneously left out the two wings from the same specimen (i.e., left and right 


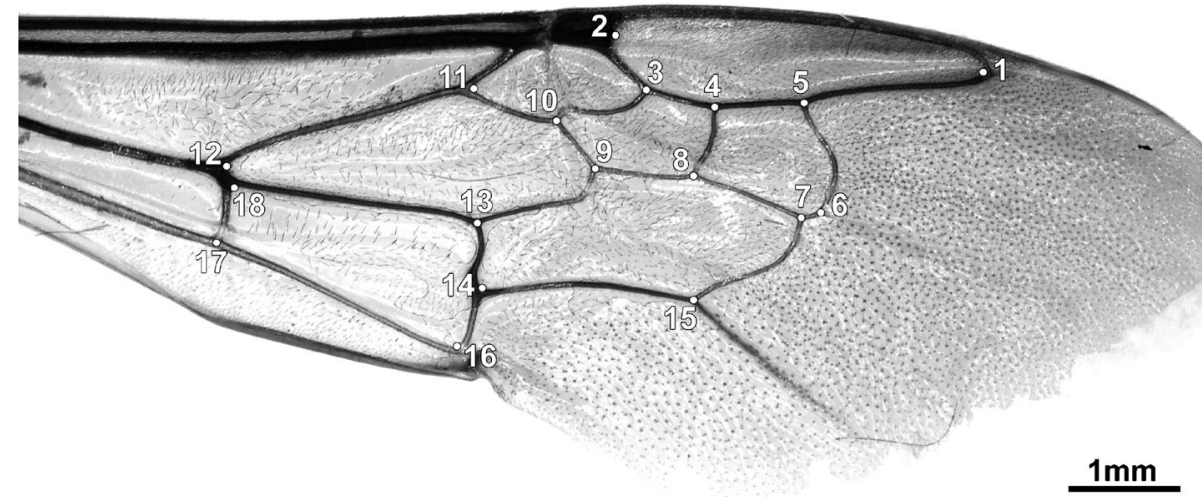

Figure 1. Wing of a Bombus terrestris haploid male with 18 landmarks.

wing) before classifying a wing in the leave-one-out procedure (Table I).

\subsubsection{Discrimination of specimens}

Assignment of wings in predictive discriminant analyses should not be considered as a unique qualitative identification. Value of the posterior probabilities could be more informative for validating the assignment to a group. Low probabilities $(<0.90)$ refer to a non- supported assignment and should be considered as dubious classification. Identification of the specimens was, therefore, redefined based on posterior probabilities of assignment of both right and left wings of the same specimen. We applied the following rule of thumb for specimen identification. A specimen was assigned to a group if both wings showed high posterior probabilities of assignment $(>0.90)$ for the same group. If one wing showed supported assignment but the second wing showed low probabilities, the specimen was

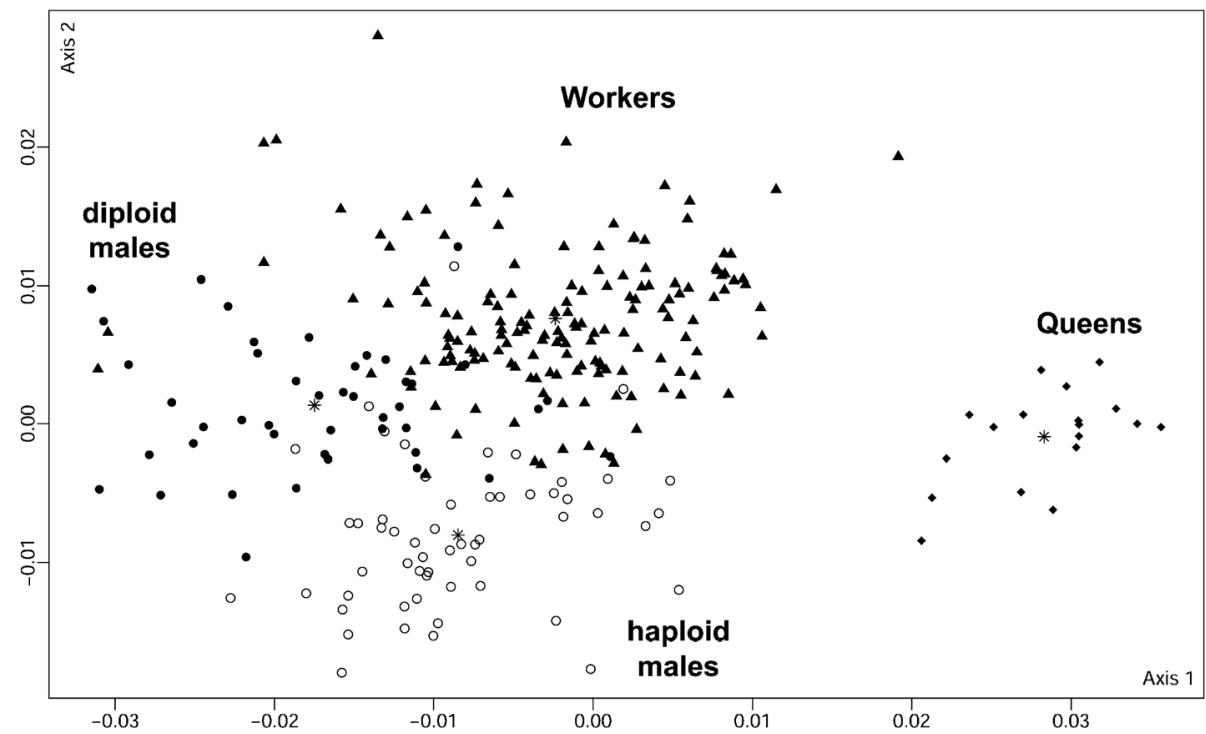

Figure 2. Ordination of the four groups of Bombus terrestris along the first two axes of the between-group principal component analysis. The four axes of the bgPCA explain $48.9 \%$ of the total variance (respectively $29.5,12,5.2$, and $2.3 \%$ ). Group means are represented by stars. Open circles : haploid males; black circles : diploid males; triangles : workers; diamonds : queens. 
Table I. Wing identification based on leave-one-out cross-validation procedure in the LDA using both wings.

\begin{tabular}{lllll}
\hline & $\mathrm{hM}$ & $\mathrm{dM}$ & $\mathrm{W}$ & $\mathrm{Q}$ \\
\hline $\mathrm{hM}$ & 39 & - & 3 & - \\
$\mathrm{dM}$ & 1 & 33 & 6 & - \\
$\mathrm{W}$ & - & 3 & 138 & - \\
$\mathrm{Q}$ & - & - & - & 18 \\
\hline
\end{tabular}

Only well-supported assignments were used $(n=241)$. Original groups are along the rows, predicted groups are along the columns

$h M$ haploid males, $d M$ diploid males, $W$ workers, $Q$ queens

assigned to the supported group. In case of divergent supported assignments, or when right and left wings both showed low probabilities, the identification was stated as dubious, and the specimen was not assigned to any group.

Separating males from females is fairly easy in bumble bees (i.e., they can be distinguished by the presence of genitalia for males and stings for females). Therefore, it is likely that in many cases one already knows the sex of a specimen and only the ploidy of the male specimen needs to be assessed. Given that wing shapes of diploid males are closer to the wing shape of workers than to the wing shape of haploid males (Figure 2, Table I), a male was identified as diploid when wings were assigned to either diploid males or workers (Table II).

Table II. Specimen identification based on leave-oneout cross-validation procedure in the LDA using both wings $(n=137)$.

\begin{tabular}{llll}
\hline & Haploid & Diploid & NA \\
\hline $\mathrm{hM}$ & 23 & 3 & 2 \\
$\mathrm{dM}$ & 0 & 21 & 1 \\
$\mathrm{~W}$ & 0 & 73 & 5 \\
$\mathrm{Q}$ & 0 & 9 & 0 \\
\hline
\end{tabular}

Original groups are along the rows, predicted ploidy along the columns. Males were identified as diploid when wings were assigned to either diploid males or workers

$h M$ haploid males, $d M$ diploid males, $W$ workers, $\underline{Q}$ queens, $N A$ dubious ploidy identification

\subsubsection{Sensitivity to the heterogeneous sample size and number of shape predictors}

Following Kovarovic et al. (2011) and Evin et al. (2013), potential bias due to heterogeneous sample sizes and number of predictors has to be taken into account in assessing the robustness of cross-validation results. As our sampling involved groups of heterogeneous size (i.e., many more worker specimens), we tested the potential effect of our sampling on the HR results.

First, PCA was used to reduce the number of shape predictors used in the LDA. Evolution of global and group HRs was calculated by sequentially increasing the number of PCs included in the LDA (Fig. S1). Because the first PCs do not always represent the most useful information for group discrimination, LDA was also calculated based on the four bgPCs (Table III).

Second, bias due to unbalanced sample size was quantified from (1) perfectly balanced groups and (2) randomized group affiliation. Balanced groups were obtained by randomly selecting 22 specimens in each group; queens (nine specimens) were excluded in this procedure. Randomized group affiliation was computed by assigning specimens to one of the four groups by chance prior to LDA calculation. Randomized group affiliations were computed both on the original and the balanced dataset. Because these procedures involve randomization, the three analyses were repeated 1000 times and summarized by average classification rate and ratio of supported classification (see rule of thumb for specimen identification described above). If overfitting due to sampling size is not playing a role, the randomized group affiliation should produce HR close to the $25 \%$ random chance baseline.

Statistical analyses were performed using the software R version 3.0.2 (2013, http://www.R-project.org/). To ensure reproducibility in our results, $\mathrm{R}$ seed was defined before calculation.

\section{RESULTS}

Mean of centroid sizes by specimen differed significantly between the four groups defined in our analyses (i.e., queens, workers, haploid males, and diploid males) as revealed by ANOVA ( $p$ value $=0$ ). Pair-wise ANOVA after Bonferroni correction (factor 6) on CS revealed significant differences between all groups ( $p$ value $=0$ for all 
Table III. Results of leave-one-out cross-validation procedures using both wings in the linear discriminant analyses on original data (LDA) and on between-groups principal components (bgLDA), and in the linear discriminant analyses on balanced groups (bLDA) and after randomized group affiliation (rLDA) ( $n=137)$.

\begin{tabular}{|c|c|c|c|c|c|c|c|c|}
\hline & \multicolumn{2}{|c|}{ LDA } & \multicolumn{2}{|c|}{ bgLDA } & \multicolumn{2}{|c|}{ bLDA } & \multicolumn{2}{|c|}{ rLDA } \\
\hline & $\mathrm{HR} \%$ & SuppID \% & $\mathrm{HR} \%$ & SuppID \% & $\mathrm{HR} \%$ & SuppID\% & $\mathrm{HR} \%$ & SuppID \% \\
\hline Global & 95 & 94 & 97 & 87 & 93 & 90 & 45 & 9 \\
\hline $\mathrm{Mh}$ & 88 & 93 & 91 & 79 & 91 & 92 & 6 & 10 \\
\hline $\mathrm{Md}$ & 86 & 95 & 100 & 77 & 90 & 90 & 4 & 11 \\
\hline W & 99 & 94 & 99 & 91 & 95 & 89 & 78 & 9 \\
\hline Q & 100 & 100 & 100 & 100 & 100 & 100 & 3 & 8 \\
\hline
\end{tabular}

For each analyses, correct classification percentage (HR\%) and percentage of supported classification (SuppID\%) are given. Mean $\mathrm{HR} \%$ and mean SuppID\% from 1000 replicates of each analysis are given for the bLDA and rLDA

$h M$ haploid males, $d M$ diploid males, $W$ workers, $Q$ queens

ANOVA) except between diploid males and workers $(p$ value $=0.358)$.

The four groups defined in our analyses (i.e., queens, workers, haploid males, and diploid males) were separated in the between-group PCA, even though clear overlap was observed between workers and males (Figure 2). In these analyses, all queens clustered together and were well separated from the other groups. The four axes of the bgPCA explained $48.9 \%$ of the total variance (respectively 29.5, 12, 5.2, and $2.3 \%$ ). Correlation coefficient between $\mathrm{CS}$ and axes of the bgPCA revealed non-influence of size on shape variables $\left(R^{2}=0.16, R^{2}=0.25, R^{2}=0.03\right.$, and $R^{2}=0.01$, respectively, for first, second, third, and fourth axes). Correlation coefficients between $\mathrm{CS}$ and axes of a PCA also revealed non-influence of size on shape variables ( $R^{2} \leq 0.2$ for all $32 \mathrm{PCs}$ ).

The same four groups were well separated from each other in the LDA. If we regard the assignment by the cross-validation, disregarding the level of support for the assignment, $92 \%$ of wings were correctly assigned to their group. The 18 queen wings showed an HR of $100 \%$. Six of the 156 worker wings were misclassified, all being assigned to diploid males (HR 96 \%). Among 56 haploid male wings, only two were misclassified as diploid male wing and six as worker wing (HR $86 \%$ ). Among 44 diploid male wings, one was misclassified as a haploid male and eight were misclassified as workers (HR of $80 \%$ ).
If, on the other hand, we only consider wellsupported assignments (i.e., with posterior probability $>0.90$; this is the case for $88 \%$ of all wings), we see that $95 \%$ of the wings were classified correctly (Table I). Having prior knowledge of the sex of the specimen, i.e., wing is known from a male, the hit ratio for assignment to the correct ploidy was $98 \%$ (39 of 40) for diploid male wings and $93 \%$ (39 of 42) for haploid male wings (Table I).

At specimen level, a well-supported identification was given for 47 of the 50 males. Except for three haploid males, the correct ploidy was assigned (Table II). The three haploid males were classified as workers. The LDA of wing shape appears to be a clear and accurate method for discrimination between haploid and diploid males. All the queens and 73 of the 78 workers had a well-supported identification and were assigned to the correct level of ploidy.

The number of shape predictors did not bias the results obtained in the LDA. Highest HRs were obtained when including all PCs in the analysis (Fig. S1). Moreover, HRs obtained in the LDA based on the bgPCs were not better than those obtained on original data (Table III). These results allow us to be confident in our classification accuracy while using all shape predictors.

However, the unbalanced sampling size between workers and other groups influenced the group HRs. Global HR, group HRs, and the 
distribution of posterior probabilities of identification calculated in the different cross-validation procedures using both wings are summarized in Table III. The randomized group affiliations computed on a balanced dataset led to mean group HR of $25 \%$ in all groups, corresponding to the random chance baseline. However, randomized group affiliations computed on the original dataset led to a highly variable group HR, ranging from mean HR of less than $10 \%$ for queens and haploid and diploid males to $78 \%$ for workers (Table III). These results suggest that, due to unequal sampling size, an average of $78 \%$ of the workers can be correctly classified by chance. As suggested by Kovarovic et al. (2011) and from the results obtained in the LDA on balanced groups (bLDA, Table III), this high random chance baseline for workers might have influenced the misclassification of the three haploid males to this larger group. In any cases, cross-validated accuracy in the LDA on original data was always better than chance.

\section{DISCUSSION}

\subsection{Haploid and diploid male production in inbred colonies}

We managed to produce diploid males in our laboratory colonies, in which approximately half of the first brood were males. The majority of those males were diploid ( $72 \%$ ), but there was a substantial number of haploid males. Even if our results are based on one colony only, they confirm similar study based on colonies reared in laboratory condition (Duchateau et al. 1994). However, these observations are unlikely to mirror wild situation. According to Roseler (1985), the $\mathrm{CO}_{2}$ narcosis can induce the laying of unfertilized eggs by queens during their first brood, which may have been the case in our breeding operation.

\subsection{Morphometric identifiers for haploid and diploid males}

Geometric morphometrics have been used previously to discriminate bees at different taxonomic levels (e.g., Aytekin et al. 2007; Francoy et al. 2008; Michez et al. 2009; De Meulemeester et al. 2012). Here, we show that bumble bee castes and diploid males can also be discriminated based on their wing shape with high HR. We argue that a morphometric identifier could be developed to provide a fast, accurate, and cost-effective evaluation of the degree of ploidy of bumble bee males, overcoming the impediments incurred by biomolecular analyses. However, we need additional experiments with wild specimens included in the dataset to extrapolate those results.

The method we describe here may be of use to the monitoring of the health and status of wild populations as well as to quality control of breeding stocks used for commercial rearing. Given that a high level of diploid males is an indication of a low level of allelic diversity, their proportion in a population may be an indication of the genetic health and/or recent trends (e.g., population decline) of wild pollinator populations (Zayed et al. 2004). Monitoring bee populations is pivotal and is becoming increasingly crucial for the evaluation of national and international targets in nature conservation and for biodiversity monitoring or ecosystem service assessments (e.g., crop pollination). Until now, most assessments of pollinator change have had to rely on species richness or distribution change estimates derived from indirect evidence (e.g., opportunistic occurrence records; Biesmeijer et al. 2006; Carvalheiro et al. 2013). Development of a morphometric identifier will provide a novel tool to rapidly evaluate both the state of populations (i.e., based on diploid/ haploid males ratio, as theoretically suggested by Zayed et al. 2004) and the impact of mitigation measures at a low cost as compared to the expertise needed and costs associated with assessments based on DNA analyses. In addition, past population trends may be inferred from analysis of museum collection specimens, for which male ploidy assessment can now be achieved non-invasively.

Furthermore, a recent study highlighted the production of diploid males in colonies from mass production facilities, indicating some level of inbreeding in industrial breeding (Maebe et al. 2014). An early morphological detection of diploid males could improve the production of the bumble bee commercial breeding.

In the near future, the morphometric identifiers can be turned into automated or semi-automated identification tools suitable for field or museum 
studies. Similar morphometrics identifiers have been developed over the past decade (MacLeod 2008 and references therein), notably DrawWing (Tofilski 2004), DAISY (O’Neill 2008), and more recently by Santana et al. (2014). Finally, morphology-based analysis will also facilitate future citizen-science bee monitoring schemes, e.g., involving smartphone or tablet-based image analysis and data recording.

\section{ACKNOWLEDGMENTS}

We wish to thank Dr. Felix Wäckers and Biobest N.V. for providing the colonies used in this study. T. De Meulemeester and J.C. Biesmeijer were supported in part by "STEP - Status and Trends of European Pollinators" (EC FP7 grant no. 244090) and by SUPER-B COST ACTION FA 1305. The study was also funded by an internal Applied-Oriented Research Grant from Naturalis Biodiversity Center. We are grateful to Collin Molenaar for help in data collection and to Prof. Menno Schilthuizen for his comments. The authors would like to thank Prof. Andrea Cardini for his valuable and constructive comments which helped to improve the manuscript.

Discrimination entre mâles de Bombus terrestris haploïdes et diploïdes (Hymenoptera; Apidae), basée sur la forme des ailes

Bourdon / déclin / morphométrie géométrique / distinction entre individus / viabilité des colonies / nervation alaire

?

Die Unterscheidung haploider und diploider Mänchen von Bombus terrestris (Hymenoptera; Apidae) anhand der Flügelform

Bombus / Verringerung / diploide Mänchen / Flügelform / geometrische Morphometrie

\section{REFERENCES}

Adams, D.C., Rohlf, F.J., Slice, D.E. (2013) A field comes of age: geometric morphometrics in the 21 st century. Hystrix 24, 7-14

Adams, D.C., Otárola-Castillo, E. (2013) Geomorph: an R package for the collection and analysis of geometric morphometric shape data. Meth. Ecol. Evol. 4, 393399

Aytekin, A.M., Terzo, M., Rasmont, P., Cagatay, N. (2007) Landmark based geometric analysis of wing shape in Sibiricobombus Vogt (Hymenoptera : Apidae : Bombus Latreille). Ann. Soc. Entomol. Fr. 43, 95-102

Biesmeijer, J.C., Roberts, S.P.M., Reemer, M., Ohlemüller, R., Edwards, M., et al. (2006) Parallel declines in pollinators and insect-pollinated plants in Britain and the Netherlands. Science 313, 351-354

Bookstein, F.L. (1991) Morphometric Tools for Landmark Data: Geometry and Biology. Cambridge University Press, Cambridge

Cameron, A.S., Lozier, J.D., Strange, J.P., Koch, J.B., Cordes, N., Solter, L.F., Griswold, T.L. (2011) Patterns of widespread decline in North American bumble bees. Proc. Natl. Acad. Sci. U. S. A. 108, 662-667

Carvalheiro, L.G., Kunin, W.E., Keil, P., Aguirre-Gutierrez, J., Ellis, W.N., et al. (2013) Species richness declines and biotic homogenization have slowed down for NWEuropean pollinators and plants. Ecol. Lett. 16, 870-878

Cournault, L., Aron, S. (2008) Rapid determination of sperm number in ant queens by flow cytometry. Insect. Soc. 55, 283-287

Crozier, R.H. (1971) Heterozygosity of sex determination in haplodiploidy. Am. Nat. 105, 399-412

Darvill, B., Ellis, J.S., Lye, G.C., Goulson, D. (2006) Population structure and inbreeding in a rare and declining bumblebee, Bombus muscorum . Mol. Ecol. 15, 601-611

Darvill, B., Lepais, O., Woodall, L.C., Goulson, D. (2012) Triploid bumblebees indicate a direct cost of inbreeding in fragmented populations. Mol. Ecol. 21 , 39883995

Davis, E.S., Murray, T.E., Fitzpatrick, N., Brown, M.J.F., Paxton, R.J. (2010) Landscape effects on extremely fragmented populations of a rare solitary bee, Colletes floralis. Mol. Ecol. 19, 4922-4935

De Meulemeester, T., Michez, D., Aytekin, A.M., Danforth, B.N. (2012) Taxonomic affinity of halictid bee fossils (Hymenoptera: Anthophila) based on geometric morphometrics analyses of wing shape. J. Syst. Paleontol. 10, 755-764

Dewulf, A., De Meulemeester, T., Dehon, M., Engel, M.S., Michez, D. (2014) A new interpretation of the bee fossil Melitta willardi Cockerell (Hymenoptera: 'Melittidae') based on geometric morphometrics of the wing. Zookeys 389, 35-48

Duchateau, M.J., Hoshiba, H., Velthuis, H.H.W. (1994) Diploid males in the bumble bee Bombus terrestris . Sex determination, sex alleles and viability. Entomol. Exp. Appl. 71 , 263-269

Ellis, J.S., Knight, M.E., Darvill, B., Goulson, D. (2006) Extremely low effective population sizes, genetic structuring and reduced genetic diversity in a threatened bumblebee species, Bombus sylvarum. Mol. Ecol. 15, 4375-4386

Estoup, A., Solignac, M., Cornuet, J.M., Goudet, J., Scholl, A. (1996) Genetic differentiation of continental and 
island populations of Bombus terrestris (Hymenoptera: Apidae) in Europe. Mol. Ecol. 5, 19-31

Evin, A., Cucchi, T., Cardini, A., Strand Vidarsdottir, U., Larson, G., Dobney, K. (2013) The long and winding road: identifying pig domestication through molar size and shape. J. Archaeol. Sci. 40, 735-743

Francoy, T.M., Wittmann, D., Drauschke, M., Muller, S., Steinhage, V., Bezerra Laure, M.A.F., De Jong, D., Gonçalves, L.S. (2008) Identification of Africanized honey bees through wing morphometrics: two fast and efficient procedures. Apidologie 39, 488-494

Gadau, J., Gerloff, C.U., Krüger, N., Chan, H., SchmidHempel, P., Wille, A., Page, R.E. (2001) A linkage analysis of sex determination in Bombus terrestris (L.) (Hymenoptera: Apidae). Heredity 87, 234-242

Herrmann, M., Trenzcek, T., Fahrenhorst, H., Engels, W. (2005) Characters that differ between diploid and haploid honey bee (Apis mellifera) drones. Genet. Mol. Res. 4, 624-641

Huberty, C.J., Olejnik, S. (2006) Applied MANOVA and Discriminant Analysis. Second Edition. New Jersey

Kovarovic, K., Aiello, L.C., Cardini, A., Lockwood, C.A. (2011) Discriminant function analyses in archaeology: are classification rates too good to be true? J. Archaeol. Sci. 38, 3006-3018

Kwon, Y.J., Saeed, S., Duchateau, M.J. (2003) Stimulation of colony initiation and colony development in Bombus terrestris by adding a male pupa: the influence of age and orientation. Apidologie 34, 429-437

Lhomme, P., Sramkova, A., Kreuter, K., Lecocq, T., Rasmont, P., Ayasse, M. (2013) A method for yearround rearing of cuckoo bumblebees (Hymenoptera: Apoidea: Bombus subgenus Psithyrus). Ann. Soc. Entomol. Fr. 49, 117-125

MacLeod, N. (2008) Automated Taxon Identification in Systematics: Theory, Approaches and Applications. CRC Press, Taylor \& Francis Group, Boca Raton

Maebe, K., Meeus, I., Maharramov, J., Grootaert, P., Rasmont, P., Smagghe, G. (2013) Microsatellite analysis in museum samples reveals inbreeding before the regression of Bombus veteranus . Apidologie 44, 188197

Maebe, K., Meeus, I., Ganne, M., Wackers, F., Smagghe, G. (2014) Scientific note on microsatellite DNA analyses revealing diploid and haploid drones in bumblebee mass breeding. Apidologie 45, 189-191

Medler, J.T. (1962) Morphometric studies on bumblebees. Ann. Entomol. Soc. Am. 55, 212-218

Michez, D., De Meulemeester, T., Nel, A., Rasmont, P., Patiny, S. (2009) New fossil evidence of the early diversification of bees: Paleohabropoda oudardi from the French Paleocene (Hymenoptera, Apidae, Anthophorini). Zool. Scr. 38, 171-181

O’Neill, M.A. (2008) DAISY: a practical computer-based tool for semi-automated species identification. In: MacLeod, N. (ed.) Automated Taxon Identification in Systematics: Theory, Approaches and Applications, pp. 101-114. CRC Press, Taylor \& Francis Group, Boca Raton
Payne, C.M., Laverty, T.M., Lachance, M.A. (2003) The frequency of multiple paternity in bumble bee (Bombus) colonies based on microsatellite DNA at the B10 locus. Insect. Soc. 50, 375-378

Potts, S.G., Biesmeijer, J.C., Kremen, C., Neumann, P., Schweiger, O., Kunin, W.E. (2010) Global pollinator declines: trends, impacts and drivers. Trends Ecol. Evol. 25, 345-353

R Development Core Team (2013) R: A language and environment for statistical computing, version 3.0.2, ISBN 3-900051-07-0. R Foundation for Statistical Computing. Vienna, Austria.

Rohlf, F.J. (1999) Shape statistics: Procrustes superimpositions and tangent spaces. J. Classif. 16, 197-223

Rohlf, F.J. (2013a) tpsUtil program, version 1.56. Department of Ecology \& Evolution, State University of New York, Stony Brook

Rohlf, F.J. (2013b) tpsDig, version 2.17. Department of Ecology and Evolution, State University of New York, Stony Brook

Rohlf, F.J. (2013c) tps-SMALL, version 1.25. Department of Ecology and Evolution, State University of New York, Stony Brook

Rohlf, F.J., Slice, D. (1990) Extensions of the Procrustes method for the optimal superimposition of landmarks. Syst. Zool. 39, 40-59

Rohlf, F.J., Marcus, L.F. (1993) A revolution in morphometrics. Trends Ecol. Evol. 8, 129-132

Roseler, P.F. (1985) A technique for year-round rearing of Bombus terrestris (Apidae, Bombini). Colonies in captivity. Apidologie 16, 165-170

Santana, F.S., Reali Costa, A.H., Truzzi, F.S., Silva, F.L., Santos, S.L., Francoy, T.M., Saraiva, A.M. (2014) A reference process for automating bee species identification based on wing images and digital image processing. Ecol. Inform. doi:10.1016/j.ecoinf.2013.12.001

Santomauro, G., Engels, W. (2002) Sexing of newly hatched live larvae of the honey bee, Apis mellifera, allows the recognition of diploid drones. Apidologie 33, 283-288

Schmid-Hempel, P., Schmid-Hempel, R., Brunner, P.C., Seeman, O.D., Allen, G.R. (2007) Invasion success of the bumblebee, Bombus terrestris, despite a drastic genetic bottleneck. Heredity 99, 414-422

Takahashi, J., Ayabe, T., Mitsuhata, M., Shimizu, I., Ono, M. (2008) Diploid male production in a rare and locally distributed bumblebee, Bombus florilegus. Insect. Soc. 55, 43-50

Tofilski, A. (2004) DrawWing, a program for numerical description of insect wings. J. Insect Sci. 4, 5pp

Van Wilgenburg, E., Driessen, G., Beukeboom, L. (2006) Single locus complementary sex determination in Hymenoptera: an unintelligent design? Frontiers Zool. 3, $1-15$

Whiting, P.W. (1933) Selective fertilization and sexdetermination in Hymenoptera. Science 78, 537538 
Winfree, R. (2010) The conservation and restoration of wild bees. Ann. N.Y. Acad. Sci. 1195, 169-197

Woyke, J. (1963) Drone larvae from fertilized eggs of the honeybee. J. Apic. Res. 2, 19-24

Zayed, A. (2009) Bee genetics and conservation. Apidologie 40, 237-262

Zayed, A., Packer, L. (2005) Complementary sex determination substantially increases extinction proneness of haplodiploid populations. Proc. Natl. Acad. Sci. U. S. A. 102, 10742-10746

Zayed, A., Roubik, D.W., Packer, L. (2004) Use of diploid male frequency data as an indicator of pollinator decline. Proc. R. Soc. B 271, 9-12

Zelditch, M.L., Swiderski, D.L., Sheets, H.D., Fink, W.L. (2004) Geometric Morphometrics for Biologists: A Primer. Elsevier Academic Press, San Diego 